\title{
A MATHEMATICAL THEORY OF QUANTUM COHOMOLOGY
}

\author{
YONGBin RuAn AND GANG Tian
}

\section{Introduction}

The notion of quantum cohomology was first proposed by the physicist C. Vafa (cf. [V]). A nice and different description was given by Witten in $[\mathrm{W}]$. The quantum cohomology plays a fundamental role in understanding mirror symmetry phenomenon of Calabi-Yau manifolds. It has also indicated many potential applications to various branches of mathematics, such as algebraic geometry. However, the whole theory is based on physical intuitions. Until recently, its rigorous mathematical foundation remained to be established. Here we announce the main results of [RT]. One of them is to prove mathematically that there is a well-defined quantum ring structure on the cohomology group of a semi-positive symplectic manifold. A symplectic manifold $(V, \omega)$ is semi-positive if for any tamed almost complex structure $J$ on $V$, there is no pseudo-holomorphic rational curves $C$ satisfying:

$$
3-n \leq \int_{C} C_{1}(V)<0
$$

To define the quantum multiplication on cohomology groups, we use topological $\sigma$ model invariants, i.e., $k$-point correlation functions of rational curves, proposed by Witten [W]. (A mathematically rigorous definition of $\mathrm{k}$-point correlation functions of rational curves, hence quantum multiplication, has been established by the first author $[R]$ for any semi-positive symplectic manifold.) The problem is to prove that this multiplication is associative. As Witten suggested, such an associativity follows from a composition law for topological $\sigma$ model invariants. Such a composition law was predicated by Witten, based on an 1+1 topological field theory axiom from physics. It is our goal to develop a mathematical theory of the composition law and of its consequences. In our paper [RT], we first define symplectic invariants of arbitrary genus using pseudo-holomorphic curves. For rational curves, those invariants are generalizations of two invariants defined by the first author in $[R]$. The main part of our paper will be devoted to a mathematical proof of the composition law of these invariants for arbitrary genus curves on semi-positive symplectic manifolds. For Grassmannians, a 
special case of the composition law was proved by Bertram [B] using algebraic geometric methods. There are many applications of the composition law. It is obvious that it can be applied to compute any $k$-point correlation function in terms of 3-point functions. In [BDW], Bertram, etc. calculated the $k$-point correlation functions for the Grassmannian $G(2, n)$. The composition law can be used to give a mathematical proof of the existence of quantum ring structures on cohomology groups of semi-positive symplectic manifolds. We will compute the quantum ring structure of complex projective space $\mathbb{C P}^{n}$ using the composition law we proved. The result coincides with what C. Vafa predicted. The composition law can be also used to construct solutions of WDVV equations, or equivalently, a family of flat connections on the total space of cohomology groups, which deform the trivial connections (cf. end of section 4). Another application is to compute the degree of the moduli space of rational curves in $\mathbb{C P}^{n}$ of fixed degree. This is a classical and difficult problem in enumerative algebraic geometry. We rigorously derive a recursion formula to compute the degree of the moduli space of rational curves. Such a formula in the case of $\mathbb{C P}^{2}$ was previously derived by Kontsevich, based on the composition law. We will also compute the degree of the moduli space of higher genus curves in $\mathbb{C P}^{2}$.

\section{Symplectic invariants}

Let $(V, \omega)$ be a semi-positive symplectic manifold, $\Sigma$ be a Riemann surface of genus $g$, and $A \in H_{2}(V, \mathbb{Z})$ with $c_{1}(V)(A) \geq 0$. Choose a generic almost complex structure $J$ on $V$, tamed by $\omega$. Let $\nu$ be a generic anti-Jlinear section of $\operatorname{Hom}\left(\pi_{1}^{*} T \Sigma, \pi_{2}^{*} T V\right)$ on $\Sigma \times V$, where $\pi_{i}$ is the projection from $\Sigma \times V$ to its $i^{\text {th }}$ factor. A section $\nu$ is said to be anti-J-linear if for any tangent vector $v$ in $T \Sigma$,

$$
\nu\left(j_{\Sigma}(v)\right)=-J(\nu(v))
$$

where $j_{\Sigma}$ is the almost complex structure on $\Sigma$. A $(J, \nu)$-perturbed holomorphic curve is a smooth map $f: \Sigma \rightarrow V$ satisfying $\left(\bar{\partial}_{J} f\right)(x)=\nu(x, f(x))$. This last equation is an inhomogeneous Cauchy-Riemann equation. We can define a mixed invariant as follows:

Fix a set of marked points $\left(x_{1}, \ldots, x_{k}\right) \in \Sigma$. Let $\alpha_{1}, \ldots, \alpha_{k}, \beta_{1}, \ldots, \beta_{l}$ be integral homology classes in $H_{*}(V, \mathbb{Z})$ satisfying:

$$
\sum_{i=1}^{k}\left(2 n-\operatorname{deg} \alpha_{i}\right)+\sum_{j=1}^{l}\left(2 n-2-\operatorname{deg} \beta_{j}\right)=2 c_{1}(V)(A)+2 n(1-g)
$$

where $g$ is the genus of $\Sigma$. Every integral homology class can be represented by a so-called pseudo-manifold. A degree $i$ pseudo-manifold is a simplical 
$i$-complex $P_{i}$ with the property that every $i-1$ simplex is the face of exactly two $i$-simplices and a continuous map $F_{i}: P_{i} \rightarrow V$. Every two such pseudomanifolds representing the same homology class are the boundary of a pseudo-manifold cobordism in the usual sense. For simplicity, we shall also use $\left\{\alpha_{i}\right\},\left\{\beta_{j}\right\}$ to denote the pseudo-manifolds representing these homology classes. Then we can choose a generic almost complex structure $J$ and a generic inhomogeneous term $\nu$ such that there are only finitely many $(J, \nu)$-perturbed holomorphic maps $f: \Sigma \rightarrow V$ satisfying: $f\left(x_{i}\right) \in\left\{\alpha_{i}\right\}$ $(1 \leq i \leq k), \quad f(\Sigma) \cap\left\{\beta_{j}\right\} \neq \emptyset(1 \leq j \leq l)$, and $f_{*}[\Sigma]=A$. For each such $f$, the set $\left\{\left(y_{1}, \ldots, y_{l}\right) ; f\left(y_{j}\right) \in\left\{\beta_{j}\right\}\right\}$ is also finite. We define the multiplicity $m(f)$ to be the algebraic sum of the elements of this set with approriate sign according to its orientation. Then, we define the mixed invariant

$$
\Phi_{(A, \omega, g)}\left(\alpha_{1}, \ldots, \alpha_{k} \mid \beta_{1}, \ldots, \beta_{l}\right)=\sum m(f) .
$$

We can prove that this number $\Phi_{(A, \omega, g)}\left(\alpha_{1}, \ldots, \alpha_{k} \mid \beta_{1}, \ldots, \beta_{l}\right)$ is independent of choices of $J, \nu$, marked points $x_{1}, \ldots, x_{k}$ in $\Sigma$, pseudo-manifolds representing $\left\{\alpha_{i}\right\},\left\{\beta_{j}\right\}$, and the conformal structure on $\Sigma$. Furthermore, the number depends only on the deformation class $\{\omega\}$ of $\omega$. Therefore we obtain a mixed invariant $\Phi_{(A,\{\omega\}, g)}\left(\alpha_{1}, \ldots, \alpha_{k} \mid \beta_{1}, \ldots, \beta_{l}\right)$, where $g$ is the genus of $\Sigma$. This invariant is nothing else but the Witten's topological $\sigma$ model invariant or $k$-point correlation function in the case $l=0$. It is also clear that $\Phi_{(A,\{\omega\}, 0)}\left(\alpha_{1}, \alpha_{2}, \alpha_{3} \mid \beta_{1}, \ldots, \beta_{l}\right)$ is just the Gromov invariant $\Phi_{(A,\{\omega\}, 0)}\left(\alpha_{1}, \alpha_{2}, \alpha_{3}, \beta_{1}, \ldots, \beta_{l}\right)$. For convenience, we would like to extend $\Phi_{(A,\{\omega\}, g)}\left(\alpha_{1}, \ldots, \alpha_{k} \mid \beta_{1}, \ldots, \beta_{l}\right)$ for any $\left\{\alpha_{i}\right\},\left\{\beta_{j}\right\}$ regardless of their degree. We simply define it to be zero unless

$$
\sum_{i=1}^{k}\left(2 n-\operatorname{deg} \alpha_{i}\right)+\sum_{j=1}^{l}\left(2 n-2-\operatorname{deg} \beta_{j}\right)=2 c_{1}(V)(A)+2 n(1-g)
$$

The invariant $\Phi_{(A,\{\omega\}, 0)}\left(\alpha_{1}, \ldots, \alpha_{k} \mid \beta_{1}, \ldots, \beta_{l}\right)$ in the case of rational curves was already defined by the first author in $[\mathrm{R}]$ for $k=0$ or $l=0$. In fact, he only defined the invariant over rational numbers since he assumed that $\alpha_{i}$ and $\beta_{j}$ can be represented by bordism classes. It is well-known that not every integral homology class can be represented by a bordism class. It was Gang Liu who pointed out to the first author that a pseudomanifold can be used in place of a bordism class to define the invariant. The use of pseudo-manifolds does not cause any extra difficulties. The construction of $\Phi_{(A,\{\omega\}, g)}\left(\alpha_{1}, \ldots, \alpha_{k} \mid \beta_{1}, \ldots, \beta_{l}\right)$ for higher genus can be processed in a similar fashion. However, for higher genus, one possible difficulty is that a sequence of $J$-holomorphic curves could degenerate to a lower genus curve. For example, a sequence of elliptic curves can degenerate to a cusp elliptic curve, which is a holomorphic sphere. Contrary 
to intuition, the dimension of moduli space will increase when the genus decreases. This used to cause a major difficulty to compactify the moduli space of pseudo-holomorphic curves of higher genus. A key observation in our work is that the phenomenon we just describe will not happen for perturbed $J$-holomorphic curves for generic nonhomogenous term. In principle, the freedom in the choice of the nonhomogenous term allows us to show that perturbed $J$-holomorphic curves only degenerate to stable curves in the sense of Deligne-Mumford. Hence, we have good control over "bad degenerations" like cusp elliptic curves. Therefore we can use perturbed $J$-holomorphic maps, instead of $J$-holomorphic maps in defining our mixed invariants for higher genus in much the same way as for genus 0 .

\section{Composition law}

Let $\mathcal{M}_{g, k}(k+2 g \geq 4)$ be the moduli space of marked Riemann surfaces $\left(\Sigma, x_{1}, \ldots, x_{k}\right)$ of genus $g$, and $\overline{\mathcal{M}}_{g, k}$ be its Deligne-Mumford compactification. We can view $\Phi_{(A,\{\omega\}, g)}$ as a constant function on $\mathcal{M}_{g, k}$. Now we let marked Riemann surfaces going to the boundry of $\overline{\mathcal{M}}_{g, k}$. We get a DeligneMumford stable curve $C$. Let us assume that $C$ consists of 2 components $C_{1}, C_{2}$ of genus $g_{1}, g_{2}$ satisfying: (1) $C_{1}$ and $C_{2}$ intersect at a double point $P$; (2) $C_{1}$ carries $m$ marked points $x_{1}, \ldots, x_{m}\left(k-2 g_{2}-2 \geq m \geq 2-2 g_{1}\right)$ and $C_{2}$ carries the rest of the marked points. Then, the composition law states that $\Phi_{(A,\{\omega\}, g)}$ can be calculated by the invariants corresponding to $C_{i}$ and contributions from the double point $P$. More precisely, we can do as follows: For simplicity, let $\left\{H_{\sigma}\right\}$ be a basis for the torsion free part of $H_{*}(V, \mathbb{Z})$. Consider the diagonal $\Delta \subset V \times V$. By the Künneth formula, the homology class of the diagonal is given by

$$
[\Delta]=\sum_{\gamma, \tau} \eta^{\gamma \tau} H_{\gamma} \otimes H_{\tau}
$$

Then we can prove

Theorem 1. Let $(V, \omega)$ be a semi-positive symplectic manifold. Fix $k$ with $(k+2 g \geq 4)$, let $\alpha_{1}, \ldots, \alpha_{k}, \beta_{1}, \ldots, \beta_{l}$ be integral homology classes, and $\left\{H_{\sigma}\right\}, \eta^{\gamma \tau}$ be given as above. Then for any $m$ with $k-2 g_{2}-2 \geq m \geq$ $2-2 g_{1}$,

$$
\begin{gathered}
\Phi_{(A,\{\omega\}, g)}\left(\alpha_{1}, \ldots, \alpha_{k} \mid \beta_{1}, \ldots, \beta_{l}\right)=\sum_{A=B_{1}+B_{2}} \sum_{j=0}^{l} \sum_{\sigma} \sum_{\gamma, \delta} \frac{1}{j !(l-j) !} \eta^{\gamma \delta} \\
\cdot \Phi_{\left(B_{1},\{\omega\}, g_{1}\right)}\left(\alpha_{1}, \ldots, \alpha_{m}, H_{\gamma} \mid \beta_{\sigma(1)}, \ldots, \beta_{\sigma(j)}\right) \\
\cdot \Phi_{\left(B_{2},\{\omega\}, g_{2}\right)}\left(\alpha_{1}, \ldots, \alpha_{m}, H_{\delta} \mid \beta_{\sigma(j+1)}, \ldots, \beta_{\sigma(l)}\right)
\end{gathered}
$$


where $\sigma$ runs over all permutations of $1, \ldots, l$.

In general, a Deligne-Mumford stable curve $C$ may have many components with complicated intersection pattern, but all intersection points are ordinary double points. Then there will be an associated composition law. Our main theorem is

Theorem 2. The composition law of the topological $\sigma$-model holds for any semi-positive symplectic manifold $\omega$ and $A$ with $c_{1}(V)(A) \geq 0$.

The precise formulation of the general composition law is rather complicated. We refer the readers to our paper [RT] for a detailed description. There is a very interesting case of this theorem. Suppose that a $k$-marked genus $g$ curve degenerates to a $k$-marked genus $g-1$ curve with a double point. Then it follows from Theorem 2 that

$$
\begin{aligned}
\Phi_{(A,\{\omega\}, g)}\left(\alpha_{1}, \ldots, \alpha_{k} \mid \beta_{1}, \ldots, \beta_{l}\right) \\
\quad=\sum_{\gamma, \tau} \eta^{\gamma \tau} \Phi_{(A,\{\omega\}, g-1)}\left(\alpha_{1}, \ldots, \alpha_{k}, H_{\gamma}, H_{\tau} \mid \beta_{1}, \ldots, \beta_{l}\right) .
\end{aligned}
$$

\section{Quantum cohomology ring}

The first application of the composition law is to establish a quantum ring structure on the cohomology of a semi-positive symplectic manifold. Put

$$
\tilde{\Phi}_{(A,\{\omega\})}\left(\alpha_{1}, \ldots, \alpha_{k}\right)=\Phi_{(A,\{\omega\}, 0)}\left(\alpha_{1}, \ldots, \alpha_{k} \mid\right)
$$

where $\alpha_{1}, \ldots, \alpha_{k}$ are integral homolgy classes. This is the Witten's $k$-point correlation function, which depends on a homology class $A$. We can also drop this condition by summing contributions over all possible $A$. So, we can formally write the $k$-point function

$$
\tilde{\Phi}_{\omega}\left(\alpha_{1}, \ldots, \alpha_{k}\right)(t)=\sum_{A} \tilde{\Phi}_{(A,\{\omega\})}\left(\alpha_{1}, \ldots, \alpha_{k}\right) e^{-t \omega(A)} .
$$

Here we encounter the problem that there may be infinitely many such $A$ who will contribute the summation. Calabi-Yau 3-folds or the $\mathbb{C P}^{2}$ blow up at 9-points are such examples. But for a monotonic symplectic manifold $V$ (i.e., $C_{1}(V)>0$ ), the Gromov-Uhlenbeck Compactness Theorem implies that this summation is finite. Let $\left\{\eta^{\sigma \tau}\right\},\left\{H_{\sigma}\right\}$ be as given in last section. It follows from Theorem 1 the composition law for $\tilde{\Phi}_{\omega}$,

$$
\begin{aligned}
& \tilde{\Phi}_{\omega}\left(\alpha_{1}, \ldots, \alpha_{k}\right)(t) \\
& \quad=\sum_{\sigma, \tau} \eta^{\sigma \tau} \tilde{\Phi}_{\omega}\left(\alpha_{1}, \ldots, \alpha_{m}, H_{\sigma}\right)(t) \tilde{\Phi}_{\{\omega\}}\left(\alpha_{m+1}, \ldots, \alpha_{k}, H_{\tau}\right)(t) .
\end{aligned}
$$


Then, we use the 3-point function to define quantum multiplication $\alpha^{*} \times_{Q}$ $\beta^{*}$ by the condition that

$$
\left(\alpha^{*} \times_{Q} \beta^{*}\right) \cup \gamma^{*}=\tilde{\Phi}_{\omega}(\alpha, \beta, \gamma)(t),
$$

where the cocycles $\alpha^{*}, \beta^{*}, \gamma^{*}$ are the Poincare duals of the cycles $\alpha, \beta, \gamma$. It is also useful to write it in terms of basis as follows:

We define

$$
f_{\alpha \beta \gamma}(t)=\tilde{\Phi}_{\omega}\left(H_{\alpha}, H_{\beta}, H_{\gamma}\right)(t)
$$

and

$$
f_{\alpha \beta}^{\delta}(t)=\eta^{\gamma \delta} f_{\alpha \beta \gamma}(t)
$$

Let $\left\{H_{\sigma}^{*}\right\}$ be the basis of $H^{*}(V, \mathbb{Z})$ which is dual to $\left\{H_{\sigma}\right\}$. Then, we define quantum multiplication

$$
H_{\alpha}^{*} \times_{Q} H_{\beta}^{*}=\sum_{\gamma} f_{\alpha \beta}^{\gamma}(t) H_{\gamma}^{*}
$$

It follows from the composition law that

$$
H_{\alpha_{1}}^{*} \times_{Q} \cdots \times_{Q} H_{\alpha_{k}}^{*}=\sum_{\gamma, \delta} \eta^{\gamma \delta} \tilde{\Phi}_{\omega}\left(H_{\alpha_{1}}, \ldots, H_{\alpha_{k}}, H_{\gamma}\right) H_{\delta}^{*}
$$

In the case of Calabi-Yau 3-folds, such a quantum multiplication is automatically associative. However, in general, the associativity is not obvious and is equivalent to the following identity:

$$
\begin{aligned}
\sum_{\sigma, \tau} \eta^{\sigma \tau} \tilde{\Phi}_{\omega}\left(H_{\alpha}, H_{\beta}, H_{\sigma}\right) \tilde{\Phi}_{\omega} & \left(H_{\tau}, H_{\gamma}, H_{\delta}\right) \\
& =\sum_{\sigma, \tau} \eta^{\sigma \tau} \tilde{\Phi}_{\omega}\left(H_{\alpha}, H_{\delta}, H_{\sigma}\right) \tilde{\Phi}_{\omega}\left(H_{\beta}, H_{\gamma}, H_{\delta}\right) .
\end{aligned}
$$

This is a direct consequence of the composition law for 4-point functions, where two different stable degenerations give two sides of the above equation. Therefore, we have

Theorem 3. The quantum multication is associative, consequently, there is a quantum ring structure on the cohomology of a semi-positive symplectic manifold $V$.

We should remark that both sides of the above equation are infinite sums, whose convergence in general remains to be checked. However, the above equation is well posed as a sequence of equations involving only finite sums. 
Example. Using the fact that there is a unique line through two points in $\mathbb{C P}^{n}$, one can show that

$$
\tilde{\Phi}_{\omega}(\mathrm{pt}, \mathrm{pt}, H)=e^{-t}
$$

where $\omega$ is the Fubini-Study class on $\mathbb{C P}^{n}$ and $H$ is a hyperplane. It implies that the quantum ring of $\mathbb{C P}^{n}$ is the quotient of $C[x]$ by the ideal generated by $x^{n+1}-e^{-t}$.

The composition law for mixed invariants also yields solutions of the WDVV equation. Let $W=H^{*}(V, \mathbb{Z}) \otimes C$. For simplicity, assume that $H^{*}(V, \mathbb{Z})$ is torsion-free. Then any $w$ in $W$ is of the form

$$
w=\sum_{j=1}^{L} t_{j} H_{j}^{*}
$$

where $L$ is the dimension of $W$. We can extend the mixed invariant $\Phi_{(A,\{\omega\}, 0)}$ to $W$ by linearity. Following E. Witten [W], one can define a generating function

$$
\Psi_{\omega}(w)=\sum_{A \in H_{2}(V, Z)} e^{-\omega(A)} \sum_{\substack{m=3 \\ w=w_{1}=\cdots=w_{m-3}}}^{\infty} \frac{1}{m !} \Phi_{(A,\{\omega\}, 0)}\left(w, w, w \mid w_{1}, \ldots, w_{m-3}\right)
$$

This function is a power series in $t_{1}, \ldots, t_{L}$. It follows from Theorem 1 in the case $k=4$ that $\Psi_{\omega}$ satisfies the WDVV equation

$$
\sum_{\sigma, \tau} \frac{\partial^{3} \Psi_{\omega}}{\partial t_{\alpha} \partial t_{\beta} \partial t_{\sigma}} \eta^{\sigma \tau} \frac{\partial^{3} \Psi_{\omega}}{\partial t_{\gamma} \partial t_{\delta} \partial t_{\tau}}=\sum_{\sigma, \tau} \frac{\partial^{3} \Psi_{\omega}}{\partial t_{\alpha} \partial t_{\gamma} \partial t_{\sigma}} \eta^{\sigma \tau} \frac{\partial^{3} \Psi_{\omega}}{\partial t_{\beta} \partial t_{\delta} \partial t_{\tau}} .
$$

If we define $A=\left\{A_{\alpha \beta}^{\gamma}\right\}$ by

$$
A_{\alpha \beta}^{\gamma}=\sum_{\tau} \eta^{\gamma \tau} \frac{\partial^{3} \Psi_{\omega}}{\partial t_{\alpha} \partial t_{\beta} \partial t_{\tau}}
$$

then $\nabla_{\epsilon}=\nabla_{0}+\epsilon A$ defines a family of connections on the tangent bundle $T W$ over $W$, where $\nabla_{0}$ is the trivial connnection on $W$. The WDVV equation is equivalent to the flatness of the connections $\nabla_{\epsilon}$. Therefore, we have a deformation of the trivial flat connection $\nabla_{0}$ by flat connections $\nabla_{\epsilon}$. 


\section{An application to enumerative geometry}

Next we give an application of our theorem to a classical problem in enumerative algebraic geometry. Let $\mathcal{N}_{n, d}$ be the moduli space of rational curves in $\mathbb{C P}^{n}$ of degree $d$. We denote by $W_{i}(2 \leq i \leq n)$ be the subvariety consisting of all those curves in $\mathcal{N}_{n, d}$ which intersect with a fixed subspace in $\mathbb{C P}^{n}$ of codimension $i$. In the case $d=1, \mathcal{N}_{n, d}$ is the Grassmannian $G(n+1,2)$, and those $W_{i}$ are just the classical schubert cycles generating the homology ring of $G(n+1,2)$. Assume that the infinitesimal deformation is unobstructed at a generic rational curve in $\mathcal{N}_{n, d}$. Then the intersection number $W_{2}^{j_{2}} \cap \cdots \cap W_{n}^{j_{n}}$ is same as the Gromov invariant

$$
\Phi_{d \ell}\left(H_{1}^{2}, \ldots, H_{j_{2}}^{2}, H_{1}^{3}, \ldots, H_{j_{n}}^{n}\right)
$$

where $\ell$ is the homology class of a line in $\mathbb{C P}^{n}, 0 \leq j_{2}, \ldots, j_{n}$ and

$$
\sum_{i=2}^{n}(i-1) j_{i}=(n+1) d+n-3 .
$$

We denote this number by

$$
\sigma_{n, d}\left(j_{2}, \ldots, j_{n}\right)
$$

We simply define $\sigma_{n, d}\left(j_{2}, \ldots, j_{n}\right)$ to be zero if $\sum_{i=2}^{n}(i-1) j_{i}$ is not $(n+1) d+n$. Then using in the case $k=4$, we can prove

Theorem 4. Assume that the infinitesimal deformation is unobstructed at a generic rational curve in $\mathcal{N}_{n, d}$. Let $\sigma_{n, d}$ be the family of intersection numbers $\left\{\sigma_{n, d}\left(j_{2}, \ldots, j_{n}\right)\right\}$. Then there are recursion formulas for computing $\sigma_{n, d}$ in terms of $\sigma_{n, 1}, \ldots, \sigma_{n, d-1}$.

We refer the readers to our paper [RT] for the explicit forms of these formulas. A special one of these recursion formulas is the following:

$$
\begin{gathered}
\sigma_{n, d}(m+1,0, \ldots, 0)-2 \sigma_{n, d}(m-1,1,0, \ldots, 0)+\sigma_{n, d}(m-2,0,1,0, \ldots, 0) \\
=\sum_{d_{1}=1}^{d-1} \sum_{j=0}^{m-2}\left(\begin{array}{c}
m-2 \\
j
\end{array}\right)\left\{\sigma_{n, d_{1}}\left(j+1,0, \ldots, j_{m_{1}+1-j}=1, \ldots, 0\right)\right. \\
\cdot \sigma_{n, d-d_{1}}\left(m-1-j, 0, \ldots, j_{n+1+j-m_{1}}=1, \ldots, 0\right) \\
-\sigma_{n, d_{1}}\left(j+2,0, \ldots, j_{m_{1}-j}=1, \ldots, 0\right) \\
\left.\cdot \sigma_{n, d-d_{1}}\left(m-2-j, 0, \ldots, j_{n+j-m_{1}}=1, \ldots, 0\right)\right\}
\end{gathered}
$$

where $m=(n+1) d+n-4$ and $m_{1}=(n+1) d_{1}+n-4$.

The number $\sigma_{n, d}((n+1) d+n, 0, \ldots, 0)$ can be interpreted as follows: Given any degree d algebraic curve $C$ in $\mathbb{C P}^{n}$, its Chow coordinate $X_{C}$ is a hypersurface in the Grassmannian manifold $G(n+1, n-1)$ and consists 
of all $(n-2)$-subspaces in $\mathbb{C P}^{n}$ which have nonempty intersection with $C$. This Chow coordinate $X_{C}$ is, uniquely up to multiplication by constants, defined by a section in $H^{0}(G(n+1, n-1), \mathcal{O}(d))$, where $\mathcal{O}(1)$ is the positive line bundle generating the Picard group of $G(n+1, n-1)$. Let $N(n, d)+1$ be the dimension of $H^{0}(G(n+1, n-1), \mathcal{O}(d))$. Then there is a subvariety in $\mathbb{C P}^{N(n, d)}$ consisting of Chow coordinates of rational (possibly singular) curves in $\mathbb{C P}^{n}$. We denote by $n_{d}$ the degree of this subvariety. If the infinitesimal deformation is unobstructed at a generic degree $d$ rational curve in $\mathbb{C P}^{n}$, then $n_{d}$ is just $\sigma_{n, d}((n+1) d+n, 0, \ldots, 0)$. Note that $n_{1}=1$. It has been a difficult problem to compute $n_{d}$ for higher degree. It follows from Theorem 4 that

Corollary 1. All the degrees $n_{d}$ can be computed.

In the case $n=2$, the assumption is automatically true. It follows from Theorem 4 that

$$
n_{d}=\frac{1}{2} \sum_{\substack{d_{1}+d_{2}=d \\ d_{1}, d_{2}>0}} \frac{d_{1} d_{2}\left(3 d d_{1} d_{2}-2 d^{2}+6 d_{1} d_{2}\right)(3 d-4) !}{\left(3 d_{1}-1\right) !\left(3 d_{2}-1\right) !} n_{d_{1}} n_{d_{2}}
$$

In particular, $n_{1}=1, n_{2}=1, n_{3}=12$. This recursion formula for computing $n_{d}$ for $\mathbb{C P}^{2}$ was first derived by Kontsevich, using the composition law previously predicted by physicists and now proved in our paper [RT].

We can also compute the degree of the moduli space of genus $g$ curves in $\mathbb{C P}^{n}$ by using the recursion formula at the end of section 3 .

\section{Outline of the proof}

Let us briefly describe our method of proving Theorems 1 and 2. Let $C_{n}$ be a sequence of stable curves with $m$-marked points, and $f_{n}: C_{n} \mapsto V$ be the corresponding pseudo-holomorphic curves. In the Deligne-Mumford compactification, $C_{n}$ degenerates to an $m$-point stable curve $C$. Geometrically, one can obtain $C$ by collapsing a disjoined union of simple closed curves. Collapsing each simple closed curve gives rise to a double point. In the case in which the $C_{n}$ are rational curves, there is a problem, i.e., the underlying complex structure really doesn't change at all. To achieve the degeneration we need to prove the composition law in this case, we take advantage of the perturbed equation and let the perturbation term degenerate along some prescribed circles in the Deligne-Mumford degeneration. By taking a subsequence, we may assume that $f_{n}$ converges to a limit map $f$ whose domain is the stable curve $C$ with some bubbles. There might be some bubbles in the vicinity of double points. Such a situation did not exist in previous compactness theorems. On each component of $C$, it 
satisfies a perturbed $J$-holomorphic equation. On each bubble, $f$ satisfies $J$-holomorphic equation. Then by counting dimensions (cf. [R]), we can show that the space of such $f$ with some bubbles will be of smaller dimension. Thus, in order to compute the invariant in terms of the perturbed $J$-holomorphic maps from the degenerate Deligne-Mumford stable curve $C$, we have to prove that any $f$ from $C$ can be deformed into a perturbed $J$-holomorphic map from a smooth marked stable curve into $V$. We also need to show that such a deformation is unique for a given inhomogeneous term and has the orientation compatible with that of $f$. In general, such a deformation is almost impossible to achieve. However, due to the freedom in the choice of inhomogeneous terms, we can prove the existence of the deformation with the required properties. This can be done by using the Implicit Function Theorem.

\section{Acknowledgement}

During the preparation of our paper, we were informed that G. Liu and D. McDuff [LM] also proved the composition law for 4-point function of rational curves independently. The first author would like to thank Kontsevich to share with him his elegant idea of deriving the recursion formula for rational curves in $\mathbb{C P}^{2}$.

\section{References}

[B] A. Bertram, Towards a schubert calculus for maps from a Riemann surfaces to a Grassmannian, in preparation.

[BDW] A. Bertram, G. Daskalopoulos and R. Wentworth, Gromov invariants for holomorphic maps from Riemann surfaces to Grassmannians, preprint.

[LM] G. Liu and D. McDuff, private communication.

[R] Y. Ruan, Topological Sigma model and Donaldson type invariants in Gromov theory, preprint.

[RT] Y. Ruan and G. Tian, A mathematical theory of quantum cohomology, in preparation.

[V] C. Vafa, Topology mirrors and quantum rings, in Essays on mirror manifolds, ed. S.-T. Yau, International Press, Hong Kong, 1992.

[W] E. Witten, Two dimensional gravity and intersection theory on moduli space, Surveys in Diff. Geom. 1 (1991), 243-310.

Dept. of Math., Univ. of Utah, Salt Lake City, UT 84112

E-mail address: ruan@math.utah.edu

Courant Institute of Math. Sci., 251 Mercer st., New York University, NY 10012

E-mail address: tiang@cims20.nyu.edu 